\title{
Tolerância Inicial de Plantas de Pinhão-Manso a Herbicidas Aplicados em Pré e Pós-Emergência ${ }^{1}$
}

\author{
Initial Tolerance of Physic Nut Plants to Pre and Post-Emergence Herbicide Application
}

\author{
ERASMO, E.A.L. ${ }^{2}$, COSTA, N.V. ${ }^{3}$, TERRA, M.A. ${ }^{3}$ e FIDELIS, R.R. ${ }^{2}$
}

\begin{abstract}
RESUMO - O presente trabalho teve por objetivo avaliar a tolerância inicial de plantas de pinhão-manso a herbicidas pré e pós-emergentes, aplicados isolados e em misturas. Foram realizados dois experimentos em condições de campo, sendo um com herbicidas pré-emergentes e outro com herbicidas pós-emergentes. Os tratamentos com os herbicidas pré-emergentes utilizados foram: atrazine $\left(3.000 \mathrm{~g} \mathrm{ha}^{-1}\right)$, diuron $\left(2.000 \mathrm{~g} \mathrm{ha}^{-1}\right)$, oxyfluorfen (720 $\left.\mathrm{g} \mathrm{ha}^{-1}\right)$, trifluralin $\left(890 \mathrm{~g} \mathrm{ha}^{-1}\right)$, pendimethalin $\left(1.250 \mathrm{~g} \mathrm{ha}^{-1}\right)$, isoxaflutole $\left(93,8 \mathrm{~g} \mathrm{ha}^{-1}\right)$, S-metolachlor $\left(1.920 \mathrm{~g} \mathrm{ha}^{-1}\right)$, atrazine+S-metolachlor $\left(1.500+960 \mathrm{~g} \mathrm{ha}^{-1}\right)$, isoxaflutole+diuron $\left(46,9+1.000 \mathrm{~g} \mathrm{ha}^{-1}\right)$, trifluralin+diuron $\left(450+1.000 \mathrm{~g} \mathrm{ha}^{-1}\right)$, além de uma testemunha sem aplicação. Os tratamentos com os herbicidas pós-emergentes foram: haloxyfop-methyl (60 $\left.\mathrm{g} \mathrm{ha}^{-1}\right)$, nicosulfuron (60 $\left.\mathrm{g} \mathrm{ha}^{-1}\right)$, sethoxydim (368 $\left.\mathrm{g} \mathrm{ha}^{-1}\right)$, fluazifop-p-butyl (125 $\left.\mathrm{g} \mathrm{ha}^{-1}\right)$, fluazifop-p-butyl (250 $\left.\mathrm{g} \mathrm{ha}^{-1}\right)$, fomesafen (125 $\left.\mathrm{g} \mathrm{ha}^{-1}\right)$, fomesafen (250 $\left.\mathrm{g} \mathrm{ha}^{-1}\right)$, fluazifop-pbutyl+fomesafen $\left(250+250 \mathrm{~g} \mathrm{ha}^{-1}\right)$, fluazifop-p-butyl+fomesafen $\left(200+250 \mathrm{~g}\right.$ ha $\left.{ }^{1}\right)$, clethodim + fenoxaprop-p-ethyl $\left(50+50 \mathrm{~g} \mathrm{ha}^{-1}\right)$, além de uma testemunha sem aplicação e outra capinada. Os tratamentos foram dispostos em delineamento experimental de blocos casualizados, com quatro repetições. No experimento com herbicidas pré-emergentes verificou-se que plantas de pinhão-manso foram tolerantes ao diuron, trifluralin, pendimethalin, isoxaflutole, $\mathrm{S}$-metolachlor e às misturas isoxaflutole+diuron e trifluralin+diuron. Com relação ao experimento com herbicidas pós-emergentes, destacaram-se o haloxyfop-methyl, sethoxydim, fluazifop-p-butyl (125 $\left.\mathrm{g} \mathrm{ha}^{-1}\right)$ e a mistura clethodim+fenoxaprop-p-ethyl.
\end{abstract}

Palavras-chave: Jatropha curcas, seletividade, biodiesel, planta daninha.

\begin{abstract}
This study aimed to evaluate the initial tolerance of physic nut plants to pre and post-emergence herbicides, applied alone and in mixtures. Thus, two experiments were conducted under field conditions, one with pre-emergence herbicides and the other with post-emergence herbicides. The treatments using the pre-emergence herbicides were: atrazine $\left(3,000 \mathrm{~g} \mathrm{ha}^{-1}\right)$, diuron $\left(2,000 \mathrm{~g} \mathrm{ha}^{-1}\right)$, oxyfluorfen $\left(720 \mathrm{~g} \mathrm{ha}^{-1}\right)$, trifluralin $\left(890 \mathrm{~g} \mathrm{ha}^{-1}\right)$, pendimethalin $(1,250 \mathrm{~g} \mathrm{ha}-1)$, isoxaflutole $\left(93,8 \mathrm{~g} \mathrm{ha}^{-1}\right)$, S-metolachlor $\left(1,920 \mathrm{~g} \mathrm{ha}^{-1}\right)$, atrazine + S-metolachlor $\left(1,500+960 \mathrm{~g} \mathrm{ha}{ }^{-1}\right)$, isoxaflutole+diuron $\left(46,9+1,000 \mathrm{~g} \mathrm{ha}^{-1}\right)$, trifluralin+diuron $\left(450+1,000 \mathrm{~g} \mathrm{ha}^{-1}\right)$ and a control without herbicide. Treatments using the post-emergence herbicides were: haloxyfop-methyl $\left(60 \mathrm{~g} \mathrm{ha}^{-1}\right)$, nicosulfuron $\left(60 \mathrm{~g} \mathrm{ha}^{-1}\right)$,

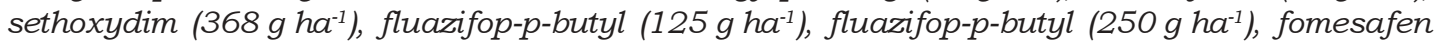
$\left(125 \mathrm{~g} \mathrm{ha}^{-1}\right)$, fomesafen $\left(250 \mathrm{~g} \mathrm{ha}^{-1}\right)$, fluazifop-p-butyl+fomesafen $\left(250+250 \mathrm{~g} \mathrm{ha}^{-1}\right)$, fluazifop-pbutyl + fomesafen $\left(200+250 \mathrm{~g} \mathrm{ha}^{-1}\right)$, clethodim + fenoxaprop-p-ethyl $\left(50+50 \mathrm{~g} \mathrm{ha}^{-1}\right)$, a control without herbicide and a hand weeded. Treatments were arranged in a randomized block design with four repetitions. It was verified that in the experiment with pre-emergence herbicides, the physic nut plants were tolerant to diuron trifluralin, pendimethalin, isoxaflutole, S-metolachlor and to the mixtures isoxaflutole+diuron and trifluralin+diuron. Regarding the experiment with post-emergence herbicides, the following showed a potential use selectivity: haloxyfop-methyl, sethoxydim, fluazifop-p-butyl (125 $\left.\mathrm{g} \mathrm{ha} \mathrm{a}^{-1}\right)$ and the mixture clethodim + fenoxaprop-p-ethyl.
\end{abstract}

Keywords: Jatropha curcas, selectivity, biodiesel, weed.

Recebido para publicação em 10.9.2008 e na forma revisada em 21.8.2009.

2 Professor Adjunto da UFT, Rua Badejós, chácaras 69 e 72 Lt.07 Zona Rural Caixa Postal 66, campus de Gurupi, $77404-970$ Gurupi-TO, <erasmolemus@uol.com.br>; ${ }^{3}$ Professor da Faculdade de Guaraí, curso de Agronomia, Av. JK, 2541 - Setor Universitário, campus de Guaraí, 77700-000 Guaraí-TO. 


\section{INTRODUÇÃO}

A produção de combustiveis a partir de óleos vegetais constitui uma estratégia importante, devido às dimensões territoriais, principalmente na região dos cerrados, e à elevada diversidade edafoclimática, além do grande número de espécies que podem ser utilizadas (Teixeira, 2005).

Entre as culturas que apresentam potencial de utilização para a produção de biocombustíveis, destaca-se a do pinhão-manso (Jatropha curcas), que tem como principal vantagem o longo ciclo produtivo, que pode chegar a 40 anos, com média de produtividade de $5 \mathrm{t} \mathrm{ha}^{-1}$ (Arruda et al., 2004; Saturnino et al., 2005; Teixeira, 2005).

Entretanto, existem vários fatores relacionados ao manejo da cultura que devem ser estudados, entre os quais destaca-se o controle de plantas daninhas, uma vez que, semelhante a outras culturas perenes, as plantas jovens de pinhão-manso podem apresentar comprometimento em seu crescimento e desenvolvimento vegetativo, bem como na produção de sementes e de óleo.

Contudo, entre os métodos de manejo das plantas daninhas utilizados em culturas perenes, o uso de herbicidas seletivos aplicados em pré ou pós-emergência das plantas daninhas pode ser uma excelente alternativa, considerando principalmente o manejo dessas espécies em extensas áreas de plantio.

Apesar de não haver ainda herbicidas seletivos registrados para a cultura do pinhãomanso no Brasil, alguns, como atrazine, diuron, clethodim, isoxaflutole, trifluralin, entre outros, que são recomendados para as culturas de mamona, mandioca e seringueira, podem apresentar seletividade na cultura do pinhão-manso, uma vez que pertencem à mesma família (Euforbiaceae), bem como os herbicidas aplicados em oleaginosas, como soja, girassol e amendoim (Rodrigues \& Almeida, 2005).

Ressalta-se ainda que devem ser realizados estudos referentes à recomendação de misturas de herbicidas para a cultura do pinhãomanso com mecanismo de ação e espectro de ação distintos. Esse recurso pode auxiliar no controle das plantas daninhas em áreas que apresentam alta variabilidade de espécies, visto que, nessa condição, a utilização de apenas um tipo de herbicida pode promover controle ineficiente das espécies de plantas daninhas.

O plantio do pinhão-manso em grandes áreas no Brasil está aumentando a cada ano, devido à crescente demanda mundial de produção de biodiesel. Entre essas áreas de plantio, destaca-se no Tocantins a Fazenda Bacaba, localizada no município de Caseara, onde atualmente existem 3 mil hectares com a cultura e com perspectiva de ampliação da área de plantio (comunicação pessoal). Contudo, existem poucas informações na literatura referentes à utilização de herbicidas isolados ou em misturas aplicados em área total na cultura do pinhão-manso para o manejo das plantas daninhas.

O presente trabalho teve por objetivo avaliar a tolerância inicial de plantas de pinhãomanso a herbicidas pré e pós-emergentes, aplicados isoladamente e em mistura.

\section{MATERIAL E MÉTODOS}

Os experimentos foram instalados na Fazenda Bacaba, localizada na rodovia TO-080, $\mathrm{km}$ 99, entre os municípios de MarianópolisTO e Caseara-TO. Foram realizados dois experimentos em condições de campo, sendo um com herbicidas pré-emergentes e outro com herbicidas pós-emergentes, aplicados na cultura do pinhão-manso.

O pinhão-manso foi semeado numa área de pastagem com Brachiaria humidicola em janeiro de 2008, a $6 \mathrm{~cm}$ de profundidade, utilizando-se plantadoura adaptada, modelo Solografic Speed Box 5000 (Baldan), no espaçamento de $3 \mathrm{~m}$ entrelinhas e $2 \mathrm{~m}$ entre plantas; cada parcela foi constituída de duas linhas, contendo seis plantas.

Antes da semeadura foi realizado o preparo convencional da área, com uma aração e duas gradagens. A adubação utilizada por cova foi de $150 \mathrm{~g}$ do formulado $5-25-15$, mais $0,04 \%$ de zinco.

Os herbicidas avaliados no experimento de pré-emergentes e de pós-emergentes são mostrados nas Tabelas 1 e 2 , respectivamente. 
Tabela 1 - Tratamentos avaliados no experimento com herbicidas pré-emergentes

\begin{tabular}{|c|c|c|c|}
\hline \multirow{2}{*}{ Produto comercial (p.c.) } & \multirow{2}{*}{ Nome comum } & Dose p.c. & Dose i.a. \\
\hline & & $\left(\right.$ g ou L ha $\left.^{-1}\right)$ & $\left(\mathrm{g} \mathrm{ha}^{-1}\right)$ \\
\hline Testemunha & --- & --- & --- \\
\hline Gesaprin 500 & atrazine & 6,0 & 3.000 \\
\hline Diuron Nortox $500 \mathrm{SC}$ & diuron & 4,0 & 2.000 \\
\hline Goal BR & oxyfluorfen & 3,0 & 720 \\
\hline Trifluralina Nortox & trifluralin & 2,0 & 890 \\
\hline Herbadox $500 \mathrm{CE}$ & pendimethalin & 2,5 & 1.250 \\
\hline Provence & isoxaflutole & 125 & 93,8 \\
\hline Dual Gold 960 & S-metolachlor & 2,0 & 1.920 \\
\hline--- & atrazine + S-metolachlor & $3,0+1,0$ & $1.500+960$ \\
\hline--- & isoxaflutole+diuron & $62,5+2,0$ & $46,9+1.000$ \\
\hline--- & trifluralin+diuron & $1,0+2,0$ & $450+1.000$ \\
\hline
\end{tabular}

Tabela 2 - Tratamentos avaliados no experimento com herbicidas pós-emergentes

\begin{tabular}{|c|c|c|c|}
\hline \multirow{2}{*}{ Produto comercial (p.c.) } & \multirow{2}{*}{ Nome comum } & Dose p.c. & Dose e.a. ou i.a. \\
\hline & & $\left(\mathrm{L} \mathrm{ha}^{-1}\right)$ & $\left(\mathrm{g} \mathrm{ha}^{-1}\right)$ \\
\hline Testemunha no limpo & --- & --- & --- \\
\hline Testemunha no sujo & --- & --- & --- \\
\hline Verdict $\mathrm{R}$ & haloxyfop-methyl & 0,5 & 60 \\
\hline Sanson $40 \mathrm{SC}$ & nicosulfuron & 1,5 & 60 \\
\hline Poast $^{1 /}$ & sethoxydim & 2,0 & 368 \\
\hline Fusilade $250 \mathrm{EW}$ & fluazifop-p-butyl & 0,5 & 125 \\
\hline Fusilade $250 \mathrm{EW}$ & fluazifop-p-butyl & 1,0 & 250 \\
\hline Flex $250^{\underline{2} /}$ & fomesafen & 0,5 & 125 \\
\hline Flex $250^{\underline{2} /}$ & fomesafen & 1,0 & 250 \\
\hline Fusilade+Flex $^{2 /}$ & fluazifop-p-butyl+fomesafen & $1,0+1,0$ & $250+250$ \\
\hline Fusilade+Flex $^{2 /}$ & fluazifop-p-butyl+fomesafen & $0,8+1,0$ & $200+250$ \\
\hline Podium $\mathrm{S}^{1 /}$ & clethodim + fenoxaprop-p-ethyl & 1,0 & $50+50$ \\
\hline
\end{tabular}

1/ À calda de pulverização foi adicionado o óleo mineral Assist $-0,5 \% \mathrm{v} \mathrm{v}^{-1}$. ${ }^{2 /}$ À calda de pulverização foi adicionado o espalhante adesivo $\mathrm{Ag}$ bem $-0,2 \% \mathrm{v} \mathrm{v}^{-1}$.

Os herbicidas de pré-emergência foram aplicados logo após a semeadura, e os de pós-emergência, 32 dias após a semeadura, quando as plantas de pinhão-manso apresentavam de seis a oito folhas e as de Brachiaria humidicola, de seis a oito perfilhos.

As aplicações dos herbicidas foram realizadas com um pulverizador costal, pressurizado a $\mathrm{CO}_{2}$ e equipado com uma barra de quatro pontas de jato plano (110UF03), distanciadas $50 \mathrm{~cm}$ entre si e com um reservatório de 2 litros, regulado para proporcionar um consumo de calda de $200 \mathrm{~L} \mathrm{ha}^{-1}$.

Para o experimento de pré-emergentes, as médias de temperatura, umidade relativa do ar e velocidade do vento no momento da aplicação dos herbicidas foram de $33,0^{\circ} \mathrm{C}$, $61,0 \%$ e $1,4 \mathrm{~m} \mathrm{~s}^{-1}$, respectivamente. No experimento de pós-emergentes, as médias de temperatura, umidade relativa do ar e velocidade do vento no momento da aplicação dos herbicidas foram de $33,1{ }^{\circ} \mathrm{C}, 67,0 \%$ e $0,6 \mathrm{~m} \mathrm{~s}^{-1}$, respectivamente.

As avaliações de fitotoxicidade nas plantas de pinhão-manso e de controle nas plantas de $B$. humidicola foram realizadas por meio de uma escala de percentual de notas visuais, em que 0 corresponde a nenhuma injúria na planta e 100 à morte das plantas (SBCPD, 1995). No experimento de pré-emergentes as 
parcelas foram capinadas frequentemente, sendo apenas avaliado o efeito da fitotoxicidade dos herbicidas sobre as plantas de pinhãomanso.

Os parâmetros utilizados para o estabelecimento das notas foram: inibição do crescimento, quantidade e uniformidade das injúrias, capacidade de rebrota das plantas e quantidade de plantas mortas.

No experimento de pré-emergentes as avaliações foram realizadas aos 12, 20, 29 e 40 dias após aplicação dos herbicidas; no de pós-emergentes, aos 8, 16, 24 e 47 dias após aplicação dos herbicidas.

Foram selecionadas quatro plantas na área central das parcelas, para avaliação dos seguintes parâmetros das plantas de pinhãomanso: altura da planta $(\mathrm{cm})$, entre o colo da planta e a folha mais alta; diâmetro do caule $(\mathrm{cm})$, a $1 \mathrm{~cm}$ do solo; e número de ramos por planta.

No final das avaliações de fitotoxicidade (47 DAA) foram coletadas duas plantas de pinhão-manso por parcela e determinada a massa seca das folhas, caule, raiz e da planta (Total), bem como foi coletado um quadro $\left(0,25 \mathrm{~m}^{2}\right)$ por parcela de plantas de
B. humidicola, para determinação da massa seca.

Os tratamentos foram dispostos em delineamento experimental de blocos casualizados, com quatro repetições. Os resultados foram submetidos à análise de variância pelo teste $\mathrm{F}$, e as médias dos tratamentos foram comparadas pelo teste LSD ( $p>0,05)$. Os dados de números de ramos por planta e de porcentagem foram transformados em $\sqrt{\mathrm{x}}+0,5$ e arco seno $\sqrt{ } \mathrm{x} / 100$, respectivamente.

\section{RESULTADOS E DISCUSSÃO}

\section{Experimento com herbicidas pré-emergentes}

Na Tabela 3, estão apresentados os resultados das avaliações visuais da fitotoxicidade nas plantas de pinhão-manso. Observou-se na primeira avaliação (12 DAA) que o diuron e o oxyflourfen foram os herbicidas que proporcionaram os maiores sintomas de injúrias nas plantas de pinhão-manso, com valores em torno de 20 e $70 \%$, respectivamente.

Aos 20 DAA, além dos herbicidas atrazine e oxyfluorfen, a mistura de atrazine + Smetolachlor apresentou sintomas de injúrias nas plantas de pinhão-manso acima de $90 \%$.

Tabela 3 - Porcentagem de fitotoxicidade em plantas de pinhão-manso, após a aplicação dos herbicidas pré-emergentes. Fazenda Bacaba, Caseara-TO, 2008

\begin{tabular}{|c|c|c|c|c|c|c|}
\hline \multirow{2}{*}{ Tratamento } & \multirow{2}{*}{$\begin{array}{c}\text { Dose } \\
\left(\mathrm{g} \text { ou } \mathrm{L} \mathrm{ha}^{-1}\right)\end{array}$} & \multicolumn{5}{|c|}{ Dia após a aplicação (DAA) } \\
\hline & & 12 & 20 & 29 & 40 & \\
\hline Testemunha & $\overline{---}$ & $0,0(0,0) \quad \mathrm{d}$ & $0,0(0,0) \quad \mathrm{d}$ & $0,0(0,0) \quad \mathrm{c}$ & $0,0(0,0)$ & $\mathrm{c}$ \\
\hline atrazine & 6,0 & $6,2(13,7) \quad b c$ & $94,0(76,5) \quad a$ & $99,0(84,3) \quad \mathrm{a}$ & $99,5(87,2)$ & $\mathrm{a}$ \\
\hline diuron & 4,0 & $21,8(23,6) \quad b$ & $2,3(8,6) \quad b c$ & $0,0(0,0) \quad \mathrm{c}$ & $0,0(0,0)$ & $\mathrm{c}$ \\
\hline oxyfluorfen & 3,0 & $76,3(61,3) \quad \mathrm{a}$ & $96,3(79,2) \quad a$ & $97,3(81,5) \quad b$ & $86,3(70.5)$ & $\mathrm{b}$ \\
\hline trifluralin & 2,0 & $3,5(10,8) \quad \mathrm{cd}$ & $1,5(6,0)$ bc & $0,0(0,0) \quad \mathrm{c}$ & $0,0(0,0)$ & $\mathrm{c}$ \\
\hline pendimethalin & 2,5 & $1,5(5,9) \quad \mathrm{cd}$ & $0,5(2,9) \quad \mathrm{cd}$ & $0,0(0,0) \quad \mathrm{c}$ & $0,0(0,0)$ & $\mathrm{c}$ \\
\hline isoxaflutole & 125,0 & $2,8(9,4) \quad \mathrm{cd}$ & $0,8(3,5) \quad \mathrm{cd}$ & $0,0(0,0) \quad \mathrm{c}$ & $0,0(0,0)$ & $\mathrm{c}$ \\
\hline S-metolachlor & 2,0 & $1,3(6,3) \quad \mathrm{cd}$ & $2,0(6,9) \quad b c$ & $0,0(0,0) \quad \mathrm{c}$ & $0,0(0,0)$ & $\mathrm{c}$ \\
\hline atrazine+S-metolachlor & $3,0+1,0$ & $3,4(10,2) \quad \mathrm{cd}$ & $96,5(79,5) \quad \mathrm{a}$ & $98,8(83,7) \quad \mathrm{a}$ & $99,0(85,1)$ & $\mathrm{a}$ \\
\hline isoxaflutole+diuron & $62,5+2,0$ & $1,5(6,1) \mathrm{cd}$ & $3,3(8,4) \quad b c$ & $0,0(0,0) \quad \mathrm{c}$ & $0,0(0,0)$ & $\mathrm{c}$ \\
\hline trifluralin+diuron & $1,0+2,0$ & $2,8(9,3) \quad \mathrm{cd}$ & $3,5(10,4) \quad b$ & $0,0(0,0) \quad \mathrm{c}$ & $0,0(0,0)$ & $\mathrm{c}$ \\
\hline $\mathrm{F}_{\text {Herbicidas }}(\mathrm{H})$ & & $15,507 * *$ & $291,082 * *$ & $4.043,206^{* *}$ & $348,715^{* *}$ & \\
\hline $\mathrm{F}_{\text {Blocos }}(\mathrm{B})$ & & $0,305^{\mathrm{ns}}$ & $1,110^{\mathrm{ns}}$ & $0,651^{\text {ns }}$ & $0,580^{\text {ns }}$ & \\
\hline d.m.s. & & 12,228 & 5,762 & 1,762 & 5,881 & \\
\hline CV (\%) & & 59,47 & 15,57 & 5,39 & 18,45 & \\
\hline
\end{tabular}

Dados originais de porcentagem foram transformados em arco seno $\sqrt{\mathrm{x}}_{\mathrm{x}} / 100$ e estão destacados entre parênteses. Médias seguidas de mesma letra na coluna não diferem estatisticamente entre si pelo teste LSD $(p<0,05)$

** significativo a $1 \%$; ${ }^{\text {ns }}$ - não significativo. 
De maneira geral, a fitotoxicidade proporcionada pelos demais herbicidas foi leve e dissipou completamente a partir dos 29 DAA. Contudo, nesse periodo, os herbicidas atrazine, oxyfluorfen e a mistura de atrazine+S-metolachlor apresentaram sintomas de injúrias de 99,0, 97,3 e 98,8\%, respectivamente.

Aos 40 DAA, as plantas que foram tratadas com o herbicida oxyfluorfen apresentaram tendência de recuperação dos sintomas de injúria causados pelo herbicida.

Com relação à fitotoxicidade provocada pela mistura de atrazine+S-metolachlor nas plantas de pinhão-manso, pode-se inferir que as injúrias foram proporcionadas pelo atrazine, mesmo sendo aplicado na metade da dose (1.500 g i.a. ha ${ }^{-1}$ ) utilizada quando aplicado individualmente, uma vez que o S-metolachlor aplicado de forma isolada (dobro da dose utilizada na mistura) provocou sintomas leves de injúrias nas plantas de pinhãomanso, as quais não foram mais evidentes a partir dos 29 DAA.

Quando se avalia o efeito dos herbicidas na altura das plantas de pinhão-manso (Tabela 4), verifica-se que atrazine e oxyfluorfen, bem como a mistura de atrazine+S-metolachlor, apresentaram diferenças estatísticas em relação à testemunha não aplicada, aos 33 DAA. Esses herbicidas apresentaram médias na ordem de 7,4, 18,6 e $7,1 \mathrm{~cm}$, respectivamente. Contudo, a partir dos 53 DAA as plantas tratadas com o oxyfluorfen demonstraram média de altura equivalente à das plantas da testemunha.

Aos 68 DAA, o atrazine isolado e a mistura dos herbicidas atrazine+S-metolachlor apresentaram as menores alturas de planta em relação à testemunha. Dessa forma, pode-se constatar que esses resultados corroboram os verificados na avaliação de fitotoxicidade (Tabela 3).

$\mathrm{Na}$ Tabela 5, pode-se verificar que os resultados do diâmetro do caule $(\mathrm{cm})$ foram similares aos obtidos para altura da planta, uma vez que o atrazine e o oxyfluorfen isolados, assim como a mistura dos herbicidas atrazine+S-metolachlor, foram os que proporcionaram as menores médias de diâmetro do caule, com valores de $0,30,1,0$ e $0,30 \mathrm{~cm}$, respectivamente, aos 33 DAA. Aos 63 DAA os herbicidas diuron e oxyfluorfen apresentaram aumento do diâmetro do caule da planta, de 72,5 e 69,8\%, respectivamente, em relação à testemunha.

Com relação ao número de ramos por planta, no geral não houve diferenças estatísticas entre os tratamentos em todos os períodos de avaliação, exceto para o atrazine e a mistura atrazine+S-metolachlor, que foram os únicos tratamentos que apresentaram as menores médias (Tabela 6).

Verificou-se que o atrazine isolado e a mistura atrazine+S-metolachlor foram os tratamentos com as maiores reduções das variáveis altura da planta, diâmetro do caule e número de ramos por planta, com valores de $70,9,46,2$ e $69,2 \%$ e $70,8,72,4$ e $69,2 \%$, respectivamente.

Todos os tratamentos que utilizaram os herbicidas diuron e S-metolachlor apresentaram reduções do número de ramos por planta, com valores inferiores a $24 \%$. Da mesma forma, o oxyfluorfen promoveu reduções apenas da altura da planta e do número de ramos por planta, com valores de 12,9 e 19,2\%, respectivamente.

Pode-se inferir que os herbicidas que apresentam seletividade às plantas de mamona, provavelmente, também podem ser seletivos para a cultura de pinhão-manso, partindo da hipótese de que pertencem à mesma família. Assim, os resultados obtidos corroboram os encontrados por Beltrão et al. (2004), quando testaram doses crescentes de diuron até 1.920 g i.a. ha ${ }^{-1}$ e observaram que não houve efeito negativo para mamoneira.

Da mesma forma, Cardoso et al. (2006) verificaram que o herbicida pendimethalin $\left(2.000 \mathrm{~g}\right.$ i.a. $\left.\mathrm{ha}^{-1}\right)$ foi seletivo à mamoneira, e que a mistura atrazine $+\mathrm{S}-$ metolachlor (Primestra Gold - 4,5 L p.c. ha ${ }^{-1}$ ) proporcionou redução da massa seca de planta em $25 \%$.

Maciel et al. (2007) verificaram que os herbicidas trifluralin (1.350 g i.a. ha $\left.\mathrm{h}^{-1}\right) \mathrm{e}$ pendimethalin (1.000 g i.a. ha-1), aplicados em pré-plantio incorporado (PPI), demonstraram boa seletividade para as variedades de mamona Íris e Al Guarany 2002. 
Tabela 4 - Média da altura $(\mathrm{cm})$ das plantas de pinhão-manso, após a aplicação dos herbicidas pré-emergentes. Fazenda Bacaba, Caseara-TO, 2008

\begin{tabular}{|c|c|c|c|c|c|}
\hline \multirow{2}{*}{ Tratamento } & Dose & \multicolumn{4}{|c|}{ Dias após a aplicação (DAA) } \\
\hline & $\left(\right.$ g ou L ha $\left.^{-1}\right)$ & 33 & 48 & 53 & 68 \\
\hline Testemunha & --- & $30,6 \mathrm{ab}$ & $38,9 \quad a b$ & $41,8 \quad a b$ & $49,0 \quad a b c c$ \\
\hline atrazine & 6,0 & $7,4 \quad d$ & $9,9 \quad \mathrm{~d}$ & $11,8 \quad b$ & 14,3 e \\
\hline diuron & 4,0 & $27,7 \quad \mathrm{ab}$ & $36,6 \quad \mathrm{ab}$ & $44,3 \quad \mathrm{a}$ & 54,6 abc \\
\hline oxyfluorfen & 3,0 & $18,6 \mathrm{c}$ & $26,2 \mathrm{c}$ & $32,8 \quad \mathrm{~b}$ & $42,7 \mathrm{~cd}$ \\
\hline trifluralin & 2,0 & $25,9 \quad b$ & $34,8 \quad a b c$ & $39,1 \quad a b$ & 44,6 bcd \\
\hline pendimethalin & 2,5 & $32,8 \quad \mathrm{a}$ & $42,5 \quad \mathrm{a}$ & $48,3 \quad a$ & 58,1 ab \\
\hline isoxaflutole & 125,0 & $26,9 \mathrm{ab}$ & $36,2 \quad a b$ & $40,8 \quad \mathrm{ab}$ & $48,1 \quad \mathrm{abcc}$ \\
\hline S-metolachlor & 2,0 & $29,3 \mathrm{ab}$ & $40,4 \quad \mathrm{ab}$ & $47,6 \quad \mathrm{a}$ & $56,5 \mathrm{ab}$ \\
\hline atrazine + S-metolachlor & $3,0+1,0$ & $7,1 \mathrm{~d}$ & $8,5 \quad \mathrm{~d}$ & $8,5 \mathrm{c}$ & $14,3 \quad \mathrm{e}$ \\
\hline isoxaflutole+diuron & $62,5+2,0$ & $25,6 \quad b$ & $31,8 \quad$ bc & $33,4 \quad b$ & $40,2 \mathrm{~d}$ \\
\hline trifluralin+diuron & $1,0+2,0$ & $28,9 \quad \mathrm{ab}$ & $39,3 \quad \mathrm{ab}$ & $48,1 \quad \mathrm{a}$ & $60,4 \quad \mathrm{a}$ \\
\hline $\mathrm{F}_{\text {Herbicidas }}(\mathrm{H})$ & & $15,027^{* *}$ & $13,959 * *$ & $14,597 * *$ & $11,288^{* *}$ \\
\hline $\mathrm{F}_{\text {Blocos }}(\mathrm{B})$ & & $1,075^{\mathrm{ns}}$ & $0,996^{\mathrm{ns}}$ & $0,897^{\mathrm{ns}}$ & $0,793^{\text {ns }}$ \\
\hline d.m.s. & & 6,630 & 9,134 & 10,489 & 13,764 \\
\hline CV (\%) & & 19,34 & 20,15 & 20,14 & 21,71 \\
\hline
\end{tabular}

Médias seguidas de mesma letra na coluna não diferem estatisticamente entre si pelo teste $\operatorname{LSD}(\mathrm{p}<0,05)$.

** significativo a $1 \%$; ${ }^{\text {ns }}$ - não significativo.

Tabela 5 - Média do diâmetro do caule $(\mathrm{cm})$ das plantas de pinhão-manso, após a aplicação dos herbicidas pré-emergentes. Fazenda Bacaba, Caseara-TO, 2008

\begin{tabular}{|c|c|c|c|c|c|}
\hline \multirow{2}{*}{ Tratamento } & \multirow{2}{*}{$\frac{\text { Dose }}{\left(\mathrm{g} \text { ou } L \mathrm{ha}^{-1}\right)}$} & \multicolumn{4}{|c|}{ Dias após a aplicação (DAA) } \\
\hline & & 33 & 48 & 53 & 68 \\
\hline Testemunha & --- & 1,53 a & $2,05 \quad a b$ & $2,20 \quad a b$ & 2,48 bc \\
\hline atrazine & 6,0 & $0,30 \mathrm{c}$ & $0,45 \mathrm{~d}$ & $0,48 \mathrm{~d}$ & $1,33 \mathrm{~cd}$ \\
\hline diuron & 4,0 & 1,43 a & $1,77 \quad a b$ & $2,06 \quad \mathrm{ab}$ & $4,28 \quad \mathrm{a}$ \\
\hline oxyfluorfen & 3,0 & $1,00 \quad b$ & $1,20 \quad \mathrm{c}$ & $1,49 \quad \mathrm{c}$ & $4,21 \quad \mathrm{a}$ \\
\hline trifluralin & 2,0 & $1,44 \quad \mathrm{a}$ & $1,70 \quad a b$ & $2,01 \quad b$ & $2,26 \quad \mathrm{bc}$ \\
\hline pendimethalin & 2,5 & $1,66 \quad \mathrm{a}$ & $2,09 \quad \mathrm{a}$ & $2,53 \quad \mathrm{a}$ & $2,79 \quad \mathrm{~b}$ \\
\hline isoxaflutole & 125,0 & $1,41 \quad \mathrm{a}$ & $1,79 \mathrm{ab}$ & $2,07 \quad a b$ & $2,41 \quad b c$ \\
\hline S-metolachlor & 2,0 & $1,59 \quad \mathrm{a}$ & $1,80 \mathrm{ab}$ & $2,17 \quad a b$ & $2,54 \mathrm{bc}$ \\
\hline atrazine + S-metolachlor & $3,0+1,0$ & $0,30 \quad \mathrm{c}$ & $0,36 \mathrm{~d}$ & $0,54 \mathrm{~d}$ & $0,69 \mathrm{~d}$ \\
\hline isoxaflutole+diuron & $62,5+2,0$ & $1,32 \quad \mathrm{a}$ & $1,62 \mathrm{bc}$ & $1,89 \mathrm{bc}$ & $2,04 \mathrm{bcd}$ \\
\hline trifluralin+diuron & $1,0+2,0$ & $1,39 \quad \mathrm{a}$ & $1,79 \quad \mathrm{ab}$ & $2,25 \quad \mathrm{ab}$ & $2,67 \quad b c$ \\
\hline $\mathrm{F}_{\text {Herbicidas }}(\mathrm{H})$ & & $15,006^{* *}$ & $13,821 * *$ & $15,854 * *$ & $4,604 * *$ \\
\hline $\mathrm{F}_{\text {Blocos }}(\mathrm{B})$ & & $1,607^{\mathrm{ns}}$ & $2,081^{\mathrm{ns}}$ & $2,010^{\mathrm{ns}}$ & $0,663^{\text {ns }}$ \\
\hline d.m.s. & & 0,357 & 0,468 & 0,498 & 1,421 \\
\hline $\mathrm{CV}(\%)$ & & 20,20 & 21,15 & 19,11 & 38,91 \\
\hline
\end{tabular}

Médias seguidas de mesma letra na coluna não diferem estatisticamente entre si pelo teste $\operatorname{LSD}(\mathrm{p}<0,05)$

** significativo a $1 \%$; ns - não significativo.

\section{Experimento com herbicidas pós-emergentes}

Na Tabela 7 são apresentadas as porcentagens de fitotoxicidade nas plantas de pinhão-manso, após a aplicação dos herbicidas pós-emergentes.
Aos 8 DAA, os herbicidas nicosulfuron, fomesafen (125 $\left.\mathrm{g} \mathrm{ha}^{-1}\right)$ e fomesafen $\left(250 \mathrm{~g} \mathrm{ha}^{-1}\right)$, bem como as misturas de fluazifopp-butyl+fomesafen $\left(250+250 \mathrm{~g} \mathrm{ha}^{-1}\right)$ e fluazifop-p-butyl+fomesafen $\left(200+250 \mathrm{~g} \mathrm{ha}^{-1}\right)$, foram os que proporcionaram os maiores 
sintomas de injúrias, com valores superiores a $40 \%$.

A partir dos 16 DAA, os herbicidas fomesafen (125 e $250 \mathrm{~g} \mathrm{ha}^{-1}$ ) e as misturas de fluazifop-p-butyl+fomesafen $\left(250+250 \mathrm{~g} \mathrm{ha}^{-1}\right) \mathrm{e}$ fluazifop-p-butyl+fomesafen $\left(200+250 \mathrm{~g} \mathrm{ha}^{-1}\right)$ mostraram tendência de redução dos sintomas de injúrias nas plantas de pinhão-manso; contudo, os valores de fitotoxicidade permaneceram acima de $28,0 \%$, até a última avaliação (47 DAA). Somente os herbicidas nicosulfuron e fluazifop-p-butyl (250 $\left.\mathrm{g} \mathrm{ha}^{-1}\right)$ apresentaram valores crescentes de fitotoxicidade durante as avaliações, atingindo valores de 99,5 e $41,3 \%$, respectivamente, aos 47 DAA.

A fitotoxicidade proporcionada pelo herbicida haloxyfop-methyl e pela mistura de clethodim+fenoxaprop-p-ethyl foi considerada leve e se dissipou completamente a partir dos 16 DAA. Da mesma maneira, os herbicidas sethoxydim e fluazifop-p-butyl (125 $\mathrm{g} \mathrm{ha}^{-1}$ ) também demonstraram fitotoxicidade leve,

Tabela 6 - Média do número de ramos das plantas de pinhão-manso, após a aplicação dos herbicidas pré-emergentes. Fazenda Bacaba, Caseara-TO, 2008

\begin{tabular}{|c|c|c|c|c|c|}
\hline \multirow{2}{*}{ Tratamento } & Dose & \multicolumn{4}{|c|}{ Dias após a aplicacão (DAA) } \\
\hline & $\left(\right.$ g ou L ha $\left.^{-1}\right)$ & 33 & 48 & 53 & 68 \\
\hline Testemunha & $\overline{---}$ & $2,4(1,7) \quad a$ & $2,4(1,7) \quad a$ & $2,6(1,8) \quad a$ & $2,6(1,8) \quad a b$ \\
\hline atrazine & 6,0 & $0,8(1,1) \quad b$ & $0,8(1,1) \quad b$ & $0,8(1,1) \quad b$ & $0,8(1,1) \quad \mathrm{c}$ \\
\hline diuron & 4,0 & $2,1(1,6) \quad \mathrm{a}$ & $2,1(1,6) \quad \mathrm{a}$ & $2,3(1,6) \quad a$ & $2,4(1,7) \quad a b$ \\
\hline oxyfluorfen & 3,0 & $2,1(1,6) \quad \mathrm{a}$ & $2,1(1,6) \quad \mathrm{a}$ & $2,1(1,6) \quad \mathrm{a}$ & $2,1(1,6) \quad a b$ \\
\hline trifluralin & 2,0 & $2,4(1,7) \quad \mathrm{a}$ & $2,4(1,7) \quad \mathrm{a}$ & $2,4(1,7) \quad \mathrm{a}$ & $2,4(1,7) \quad a b$ \\
\hline pendimethalin & 2,5 & $2,8(1,8) \quad \mathrm{a}$ & $2,8(1,8) \quad \mathrm{a}$ & $2,8(1,8) \quad \mathrm{a}$ & $3,0(1,9) \quad a$ \\
\hline isoxaflutole & 125,0 & $2,1(1,6) \quad \mathrm{a}$ & $2,1(1,6) \quad \mathrm{a}$ & $2,1(1,6) \quad \mathrm{a}$ & $2,1(1,6) \quad a b$ \\
\hline S-metolachlor & 2,0 & $2,3(1,6) \quad \mathrm{a}$ & $2,3(1,6) \quad \mathrm{a}$ & $2,3(1,7) \quad \mathrm{a}$ & $2,3(1,7) \quad a b$ \\
\hline atrazine + S-metolachlor & $3,0+1,0$ & $0,8(1,1) \quad b$ & $0,8(1,1) \quad b$ & $0,8(1,1) \quad b$ & $0,8(1,1) \quad \mathrm{c}$ \\
\hline isoxaflutole+diuron & $62,5+2,0$ & $2,0(1,6) \quad \mathrm{a}$ & $2,0(1,6) \quad \mathrm{a}$ & $2,0(1,6) \quad \mathrm{a}$ & $2,0(1,6) \quad b$ \\
\hline trifluralin+diuron & $1,0+2,0$ & $2,3(1,7) \quad \mathrm{a}$ & $2,3(1,7) \quad \mathrm{a}$ & $2,3(1,7) \quad \mathrm{a}$ & $2,3(1,7) \quad a b$ \\
\hline $\mathrm{F}_{\text {Herbicidas }}(\mathrm{H})$ & & $5,741 * *$ & $5,741 * *$ & $5,909 * *$ & $6,304 * *$ \\
\hline $\mathrm{F}_{\text {Blocos }}(\mathrm{B})$ & & $3,637 *$ & $3,637^{*}$ & $4,894 * *$ & $5,150 * *$ \\
\hline d.m.s. & & 0,285 & 0,285 & 0,290 & 0,282 \\
\hline $\mathrm{CV}(\%)$ & & 12,70 & 12,70 & 12,83 & 12,37 \\
\hline
\end{tabular}

Os dados originais foram transformados em $\sqrt{x}_{\mathrm{x}}+0,5$ e estão destacados entre parênteses.

Médias seguidas de mesma letra na coluna não diferem estatisticamente entre si pelo teste $\operatorname{LSD}(\mathrm{p}<0,05)$.

Tabela 7 - Porcentagem de fitotoxicidade em plantas de pinhão-manso, após a aplicação dos herbicidas pós-emergentes. Fazenda Bacaba, Caseara-TO, 2008

\begin{tabular}{|c|c|c|c|c|c|}
\hline \multirow{2}{*}{ Tratamento } & Dose & \multicolumn{4}{|c|}{ Dias após a aplicação (DAA) } \\
\hline & $\left(\mathrm{L} \mathrm{ha}^{-1}\right)$ & 8 & 16 & 24 & 47 \\
\hline Testemunha & --- & $0,0(0,0) \quad \mathrm{e}$ & $0,0(0,0) \quad \mathrm{d}$ & $0,0(0,0) \quad \mathrm{d}$ & $0,0(0,0) \quad \mathrm{d}$ \\
\hline haloxyfop-methyl & 0,5 & $4,3(9,9) \quad \mathrm{d}$ & $0,5(2,0) \quad d$ & $0,0(0,0) \quad \mathrm{d}$ & $0,0(0,0) \quad \mathrm{d}$ \\
\hline nicosulfuron & 1,5 & $42,5(40,7) \quad b$ & $75,0(60,2) \quad a b$ & $81,3(64,4) \quad a$ & $99,5(87,1) \quad$ a \\
\hline sethoxydim & 2,0 & $1,3(18,8) \quad \mathrm{c}$ & $2,5(4,6) \quad d$ & $0,0(0,0) \quad d$ & $0,0(0,0) \quad d$ \\
\hline fluazifop-p-butyl & 0,5 & $5,5(11,6) \quad \mathrm{cd}$ & $2,5(6,4) \quad d$ & $1,3(3,2) \quad d$ & $0,0(0,0) \quad d$ \\
\hline fluazifop-p-butyl & 1,0 & $3,8(9,7) \quad \mathrm{d}$ & $3,8(9,7) \quad \mathrm{d}$ & $7,5(11,4) \quad d$ & $41,3(33,4) \quad \mathrm{c}$ \\
\hline fomesafen & 0,5 & $82,5(65,5) \quad a$ & $53,8(46,9) \quad \mathrm{c}$ & $50,0(44,8) \quad \mathrm{c}$ & $45,0(41,9) \quad b c$ \\
\hline fomesafen & 1,0 & $90,0(72,3) \quad a$ & $60,0(50,8) \quad b c$ & $58,8(50,2) \quad$ bc & $56,5(48,9) \quad$ bc \\
\hline fluazifop-p-butyl+fomesafen & $1,0+1,0$ & $85,0(67,4) \quad \mathrm{a}$ & $71,3(57,6) \quad a b$ & $47,5(43,3) \quad \mathrm{c}$ & $28,8(29,6) \quad \mathrm{c}$ \\
\hline fluazifop-p-butyl+fomesafen & $0,8+1,0$ & $88,8(70,9) \quad \mathrm{a}$ & $81,3(65,0) \quad \mathrm{a}$ & $71,3(58,1) \quad a b$ & $67,5(56,3) \quad b$ \\
\hline clethodim + fenoxaprop-p-ethyl & 1,0 & $5,3(11,4) \quad \mathrm{cd}$ & $0,0(0,0) \quad \mathrm{d}$ & $0,0(0,0) \quad \mathrm{d}$ & $0,0(0,0) \quad \mathrm{d}$ \\
\hline $\mathrm{F}_{\text {Herbicidas }}(\mathrm{H})$ & & $104,710^{* *}$ & $62,627 * *$ & $39,550 * *$ & $17,025^{* *}$ \\
\hline $\mathrm{F}_{\text {Blocos }}(\mathrm{B})$ & & $2,113^{\mathrm{ns}}$ & $0,653^{\mathrm{ns}}$ & $1,283^{\mathrm{ns}}$ & $1,239^{\mathrm{ns}}$ \\
\hline d.m.s. & & 8,252 & 10,167 & 12,301 & 20,846 \\
\hline $\mathrm{CV}(\%)$ & & 16,63 & 25,52 & 34,01 & 53,41 \\
\hline
\end{tabular}

Os dados originais foram transformados em arco seno $\sqrt{\mathrm{x}} / 100$ e estão destacados entre parênteses. Médias seguidas de mesma letra na coluna não diferem estatisticamente entre si pelo teste LSD $(\mathrm{p}<0,05)$.

** significativo a $1 \%$; ${ }^{\text {ns }}$ - não significativo. 
que se dissipou completamente aos 24 e 47 DAA, respectivamente.

As médias de altura da planta, diâmetro do colmo e número de ramos das plantas de pinhão-manso, após 47 dias da aplicação dos herbicidas pós-emergentes, estão evidenciadas na Tabela 8. Ao analisar a altura das plantas, pode-se verificar que os herbicidas nicosulfuron e fluazifop-p-butyl+fomesafen $\left(200+250 \mathrm{~g} \mathrm{ha}^{-1}\right)$ foram os que apresentaram as menores médias, com valores de 7,9 e $18,9 \mathrm{~cm}$, respectivamente, porém somente o nicosulfuron diferiu estatisticamente em relação às testemunhas. A maior altura de plantas foi constatada no tratamento correspondente à mistura clethodim + fenoxapropp-ethyl, com média de $32,0 \mathrm{~cm}$, a qual não diferiu dos tratamentos sethoxydim, haloxyfopmethyl e fluazifop-p-butyl (125 e $250 \mathrm{~g} \mathrm{ha}^{-1}$ ), diferindo estatisticamente das testemunhas. A convivência com as plantas de $B$. humidicola não promoveu redução da altura das plantas de pinhão-manso, quando comparada à testemunha no limpo.

Com relação ao diâmetro do caule, apenas os tratamentos nicosulfuron, fomesafen (125 e $250 \mathrm{~g} \mathrm{ha}^{-1}$ ) e a mistura fluazifop-pbutyl+ fomesafen $\left(200+250 \mathrm{~g} \mathrm{ha}^{-1}\right)$ foram os que apresentaram as menores médias, diferindo estatisticamente quando comparados com a testemunha no limpo. Quanto ao número de ramos por planta, os tratamentos não diferiram estatisticamente em relação às testemunhas, com exceção do nicosulfuron, no qual foi verificada a menor média.

Na Tabela 9 estão evidenciados os dados de porcentagem de controle das plantas de B. humidicola, após aplicação dos herbicidas pós-emergentes. Pode-se observar que apenas os tratamentos com fomesafen em ambas as doses não exerceram controle das plantas de $B$. humidicola; esse efeito já era esperado, uma vez que o fomesafen é recomendado no controle de plantas daninhas de folha larga (Rodrigues \& Almeida, 2005). Contudo, a partir dos 16 DAA os herbicidas haloxyfop-methyl, nicosulfuron, sethoxydim, fluazifop-p-butyl (125 e $250 \mathrm{~g} \mathrm{ha}^{-1}$ ) e as misturas fluazifop-p-butyl+fomesafen $(250+250$ e 200+250 $\mathrm{g} \mathrm{ha}^{-1}$ ) e clethodim+ fenoxaprop-pethyl apresentaram médias de controle acima de $70 \%$; entretanto, aos $47 \mathrm{DAA}$, apenas os herbicidas nicosulfuron, sethoxydim, fluazifopp-butyl (125 e $\left.250 \mathrm{~g} \mathrm{ha}^{-1}\right)$ e a mistura clethodim+ fenoxaprop-p-ethyl obtiveram controle das plantas de $B$. humidicola superior a $88,5 \%$.

Tabela 8 - Médias de altura da planta, diâmetro do caule e número de ramos das plantas de pinhão-manso, após 47 dias da aplicação dos herbicidas pós-emergentes. Fazenda Bacaba, Caseara-TO, 2008

\begin{tabular}{|c|c|c|c|c|}
\hline \multirow[b]{2}{*}{ Tratamento } & Dose & \multicolumn{3}{|c|}{ 36 Dias após a aplicação (DAA) } \\
\hline & $\left(\mathrm{L} \mathrm{ha}^{-1}\right)$ & Altura $(\mathrm{cm})$ & $\begin{array}{c}\text { Diâmetro do caule } \\
(\mathrm{cm})\end{array}$ & $\mathrm{N}^{\mathrm{o}}$ ramos por planta \\
\hline Testemunha no limpo & --- & 24,8 bcd & 1,35 abcd & $2,1(1,6) \quad a b$ \\
\hline Testemunha no sujo & --- & 23,1 bcd & 1,00 defgh & $1,5(1,4) \quad b$ \\
\hline haloxyfop-methyl & 0,5 & $28,3 \quad \mathrm{ab}$ & $1,53 \mathrm{ab}$ & $1,9(1,5) \quad a b$ \\
\hline nicosulfuron & 1,5 & $7,9 \quad \mathrm{e}$ & $0,45 \mathrm{~h}$ & $0,5(0,1) \quad \mathrm{c}$ \\
\hline sethoxydim & 2,0 & $28,5 \quad \mathrm{ab}$ & 1,48 abc & $1,9(1,5) \quad a b$ \\
\hline fluazifop-p-butyl & 0,5 & 26,2 abc & 1,25 bcdef & $2,2(1,6) \quad a$ \\
\hline fluazifop-p-butyl & 1,0 & 25,8 abc & 1,28 bcde & $2,0(1,6) \quad a b$ \\
\hline fomesafen & 0,5 & 23,9 bcd & 0,88 fgh & $1,7(1,5) \quad a b$ \\
\hline fomesafen & 1,0 & $20,9 \mathrm{~cd}$ & 0,90 efgh & $2,1(1,6) \quad a b$ \\
\hline fluazifop-p-butyl+fomesafen & $1,0+1,0$ & $21,2 \mathrm{~cd}$ & 1,13 cdefgh & $2,4(1,7) \quad a$ \\
\hline fluazifop-p-butyl+fomesafen & $0,8+1,0$ & $18,9 \mathrm{~d}$ & $0,77 \mathrm{gh}$ & $2,0(1,6) \quad a b$ \\
\hline clethodim + fenoxaprop-p-ethyl & 1,0 & $32,0 \quad \mathrm{a}$ & $1,73 \quad \mathrm{a}$ & $2,1(1,6) \quad a b$ \\
\hline $\mathrm{F}_{\text {Herbicidas }}(\mathrm{H})$ & & $7,976^{* *}$ & $6,924 * *$ & $4,073^{* *}$ \\
\hline $\mathrm{F}_{\text {Blocos }}(\mathrm{B})$ & & $3,769^{\mathrm{ns}}$ & $2,555^{\mathrm{ns}}$ & $2,789^{\mathrm{ns}}$ \\
\hline d.m.s. & & 6,235 & 0,394 & 0,697 \\
\hline CV $(\%)$ & & 18,48 & 23,99 & 25,93 \\
\hline
\end{tabular}

${ }^{1 /}$ Os dados originais foram transformados em arco seno $\sqrt{ } \mathrm{x}+0,5$ e estão destacados entre parênteses. Médias seguidas de mesma letra na coluna não diferem estatisticamente entre si pelo teste LSD $(\mathrm{p}<0,05)$

** significativo a $1 \%$; ${ }^{\text {ns }}$ - não significativo. 
Observou-se efeito antagônico do fomesafen sobre o fluazifop-p-butyl, visto que o controle das plantas de $B$. humidicola com a mistura foi inferior ao obtido pelos tratamentos isolados de fluazifop-p-butyl.

Com relação ao acúmulo de massa seca pelas plantas de pinhão-manso, aos 47 DAA (Tabela 10), pode-se verificar que os herbicidas nicosulfuron e fomesafen, em ambas as doses, foram os que promoveram os menores acúmulos de massa seca, principalmente na folha, no caule e no somatório (folha, caule e raiz), com diferenças estatísticas em comparação com a testemunha no limpo.

Quanto ao acúmulo de massa seca das plantas de $B$. humidicola, os dados corroboram os obtidos na Tabela 9 , em que os maiores acúmulos correspondem aos tratamentos que

Tabela 9 - Porcentagem de controle de plantas de B. humidicola, após aplicação dos herbicidas pós-emergentes. Fazenda Bacaba, Caseara-TO, 2008

\begin{tabular}{|c|c|c|c|c|c|c|}
\hline \multirow{2}{*}{ Tratamento } & Dose & \multicolumn{5}{|c|}{ Dias após a aplicação (DAA) } \\
\hline & $\left(\mathrm{L} \mathrm{ha}^{-1}\right)$ & 8 & 16 & 24 & 47 & \\
\hline Testemunha & $\overline{---}$ & $0,0(0,0) \quad f$ & $0,0(0,0) \quad \mathrm{d}$ & $0,0(0,0) \quad \mathrm{c}$ & $\overline{0,0(0,0) \quad \mathrm{d}}$ & $\bar{d}$ \\
\hline haloxyfop-methyl & 0,5 & $38,8(38,3) \quad$ bcd & $82,5(66,3) \quad a b c$ & $67,8(56,8) \quad b$ & $69,8(59,2) \quad b$ & $\mathrm{bc}$ \\
\hline nicosulfuron & 1,5 & $27,5(31,1) \quad d$ & $73,8(59,8) \quad \mathrm{c}$ & $83,8(66,8) \quad a b$ & $96,8(80,1) \quad$ a & $\mathrm{a}$ \\
\hline sethoxydim & 2,0 & $32,5(34,3) \quad \mathrm{cd}$ & $77,5(61,8)$ bc & $83,8(66,9) \quad a b$ & $92,3(75,0) \quad \mathrm{a}$ & $\mathrm{a}$ \\
\hline fluazifop-p-butyl & 0,5 & $36,3(36,9) \quad \mathrm{cd}$ & $76,3(61,4) \quad b c$ & $86,3(68,4) \quad a b$ & $88,5(71,6) \quad \mathrm{a}$ & $\mathrm{ab}$ \\
\hline fluazifop-p-butyl & 1,0 & $53,8(47,4) \quad a b$ & $88,8(70,9) \quad a$ & $91,5(73,6) \quad \mathrm{a}$ & $97,0(80,2) \quad \mathrm{a}$ & $\mathrm{a}$ \\
\hline fomesafen & 0,5 & $0,0(0,0) \quad \mathrm{f}$ & $0,0(0,0) \quad \mathrm{d}$ & $0,0(0,0) \quad \mathrm{c}$ & $0,0(0,0) \quad \mathrm{d}$ & $\mathrm{d}$ \\
\hline fomesafen & 1,0 & $7,5(13,8) \quad \mathrm{e}$ & $0,0(0,0) \quad d$ & $0,0(0,0) \quad \mathrm{c}$ & $0,0(0,0) \quad \mathrm{d}$ & $\mathrm{d}$ \\
\hline fluazifop-p-butyl+fomesafen & $1,0+1,0$ & $63,8(53,1) \quad a$ & $88,0(69,8) \quad a b$ & $88,8(70,5) \quad a$ & $62,5(52,5) \quad \mathrm{c}$ & $\mathrm{c}$ \\
\hline fluazifop-p-butyl+fomesafen & $0,8+1,0$ & $68,8(56,2) \quad a$ & $87,5(70,0) \quad a b$ & $68,8(56,4) \quad b$ & $61,3(52,4) \quad c$ & $\mathrm{c}$ \\
\hline clethodim + fenoxaprop-p-ethyl & 1,0 & $42,5(40,6)$ bc & $90,0(72,1) \quad \mathrm{a}$ & $96,3(79,2) \quad a$ & $96,3(79,9) \quad \mathrm{a}$ & $\mathrm{a}$ \\
\hline$\overline{F_{\text {Herbicidas }}(\mathrm{H})}$ & & $36,419^{* *}$ & $109,297 * *$ & $47,657^{* *}$ & $41,141^{* *}$ & \\
\hline $\mathrm{F}_{\text {Blocos }}(\mathrm{B})$ & & $3,083^{*}$ & $1,067^{\mathrm{ns}}$ & $0,878^{\mathrm{ns}}$ & $2,272^{\mathrm{ns}}$ & \\
\hline d.m.s. & & 9,310 & 8,660 & 13,440 & 15,020 & \\
\hline CV $(\%)$ & & 20,17 & 12,39 & 19,00 & 21,02 & \\
\hline
\end{tabular}

Os dados originais foram transformados em arco seno $\sqrt{\mathrm{X}}_{\mathrm{x}} / 100$ e estão destacados entre parênteses. Médias seguidas de mesma letra na coluna não diferem estatisticamente entre si pelo teste LSD $(\mathrm{p}<0,05)$.

** significativo a $1 \%$; ${ }^{*}$ significativo a $5 \%$; ${ }^{\text {ns }}$ - não significativo.

Tabela 10 - Médias de massa seca (g) das plantas de pinhão-manso e de B. humidicola, após 47 dias da aplicação dos herbicidas pósemergentes. Fazenda Bacaba, Caseara-TO, 2008

\begin{tabular}{|c|c|c|c|c|c|c|}
\hline \multirow{2}{*}{ Tratamento } & \multirow{2}{*}{$\begin{array}{c}\text { Dose } \\
\left(\mathrm{L} \mathrm{h} \mathrm{h}^{-1}\right)\end{array}$} & \multicolumn{4}{|c|}{ Pinhão-manso } & \multirow{2}{*}{ B. humidicula } \\
\hline & & Folha & Caule & Raiz & Total & \\
\hline Testemunha no limpo & --- & $3,6 \quad a b$ & $15,5 \mathrm{ab}$ & 2,4 abc & $21,4 \quad a b$ & --- \\
\hline Testemunha no sujo & --- & 2,0 bcde & 10,9 bcd & 2,2 abcd & 15,1 bcde & $4,5 \mathrm{~b}$ \\
\hline haloxyfop-methyl & 0,5 & 3,0 abcd & 13,2 abc & 2,1 abcd & $18,4 \quad a b c$ & 1,9 de \\
\hline nicosulfuron & 1,5 & $0,7 \mathrm{e}$ & $2,7 \quad \mathrm{e}$ & $0,7 \quad \mathrm{~d}$ & $4,1 \quad \mathrm{e}$ & 2,4 de \\
\hline sethoxydim & 2,0 & $4,3 \quad \mathrm{a}$ & $15,7 \quad \mathrm{ab}$ & $3,4 \quad \mathrm{ab}$ & $27,7 \quad a b$ & 2,4 cde \\
\hline fluazifop-p-butyl & 0,5 & 3,4 abcd & $14,9 \quad \mathrm{ab}$ & $3,2 \quad a b$ & $21,6 \quad a b$ & $1,2 \mathrm{e}$ \\
\hline fluazifop-p-butyl & 1,0 & 2,4 abcde & 11,9 bcd & 2,1 abcd & 16,4 bcd & 2,5 cde \\
\hline fomesafen & 0,5 & $1,1 \mathrm{de}$ & 5,5 cde & $1,1 \mathrm{~cd}$ & 7,7 cde & $6,1 \quad \mathrm{a}$ \\
\hline fomesafen & 1,0 & 1,2 cde & 4,5 de & $1,1 \quad \mathrm{~cd}$ & 6,8 de & $4,0 \quad b c$ \\
\hline fluazifop-p-butyl+fomesafen & $1,0+1,0$ & 3,4 abc & 10,5 bcd & 1,9 bcd & 15,9 bcd & 3,4 bcd \\
\hline fluazifop-p-butyl+fomesafen & $0,8+1,0$ & 1,7 bcde & 6,1 cde & $1,3 \mathrm{~cd}$ & 9,2 cde & $4,1 \quad b$ \\
\hline clethodim + fenoxaprop-p-ethyl & 1,0 & $3,6 \quad a b$ & $20,4 \quad \mathrm{a}$ & $3,7 \quad \mathrm{a}$ & $27,7 \quad \mathrm{a}$ & $1,5 \mathrm{e}$ \\
\hline $\mathrm{F}_{\text {Herbicidas }}(\mathrm{H})$ & & $2,211 * *$ & $3,941 * *$ & $2,825 * *$ & $3,682 * *$ & $7,467^{* *}$ \\
\hline $\mathrm{F}_{\text {Blocos }}(\mathrm{B})$ & & $1,953^{\mathrm{ns}}$ & $2,741^{\mathrm{ns}}$ & $1,202^{\text {ns }}$ & $2,358^{\text {ns }}$ & $1,663^{\mathrm{ns}}$ \\
\hline d.m.s. & & 2,290 & 7,782 & 1,611 & 11,037 & 1,571 \\
\hline CV $(\%)$ & & 62,90 & 49,28 & 53,22 & 49,11 & 35,38 \\
\hline
\end{tabular}

Médias seguidas de mesma letra na coluna não diferem estatisticamente entre si pelo teste LSD $(p<0,05)$.

** significativo a $1 \%$; ${ }^{\text {ns }}$ - não significativo. 
apresentaram controle ineficiente, destacando-se os herbicidas fomesafen nas duas doses utilizadas e a mistura fluazifop-pbutyl+fomesafem nas doses de 250+250 e 200+250 $\mathrm{g} \mathrm{ha}^{-1}$.

Assim, com base nos resultados do presente trabalho, pode-se concluir que os herbicidas pré-emergentes diuron, trifluralin, pendimethalin, isoxaflutole, S-metolachlor e as misturas isoxaflutole+diuron e trifluralin+ diuron apresentaram potencial de utilização na cultura do pinhão-manso. Com relação aos herbicidas pós-emergentes, destacaram-se o haloxyfop-methyl, sethoxydim, fluazifop-p-butyl (125 $\left.\mathrm{g} \mathrm{ha}^{-1}\right)$ e a mistura clethodim+fenoxapropp-ethyl.

Contudo, para ser recomendado de forma definitiva e indiscriminada para uma determinada cultura, um herbicida deve demonstrar seletividade aos cultivares mais comuns dessa cultura (Velini et al., 2000). Assim, devem ser realizados mais estudos referentes à seletividade de herbicidas na cultura do pinhão-manso, conduzidos até a produção, incluindo-se na avaliação o parâmetro de qualidade do óleo.

Existem várias moléculas disponíveis no mercado nacional que devem ser testadas, em diferentes doses e estádios vegetativos da planta. Além disso, a cultura do pinhão-manso ainda não possui um padrão genético estabelecido dentro de programas de melhoramento, o que acarreta grande variabilidade e desuniformidade de plantas em condições de campo. Esse fato pode influenciar significativamente os resultados das respostas de tolerância das plantas à aplicação de determinado herbicida, após o processo de melhoramento vegetal.

\section{LITERATURA CITADA}

ARRUDA, F. P. et al. Cultivo de pinhão-manso (Jatropha curcas) como alternativa para o semi-árido nordestino. R. Bras. Oleag. Fibrosas, v. 8, n. 1, p. 789-799, 2004.

BELTRÃO. N.E. M. et al. Herbicidas diuron e pendimethalin na cultura da mamona, cultivo solteiro, no sudoeste da Bahia. In: CONGRESSO BRASILEIRO DE MAMONA, ENERGIA E SUSTENTABILIDADE, 1. 2004, Campina Grande. Anais... Campina Grande: 2004. CD-ROM

CARDOSO, G. D. et al. Estudo preliminar de seletividade de herbicidas à cultura da mamona. In: CONGRESSO BRASILEIRO DE MAMONA, CENÁRIO ATUAL E PERSPECTIVAS, 2., 2006, Aracaju. Anais... Aracaju: 2006. CD-ROM

MACIEL, C. D. G. et al. Seletividade de herbicidas em cultivares de mamona. R. Oleag. Fibrosas, v. 11, n. 1, p. $47-54,2007$

RODRIGUES, B. N.; ALMEIDA, F. S. Guia de herbicidas. 5.ed. Londrina: 2005. 592 p.

SATURNINO, H. M. et al. Cultura do pinhão-manso (Jatrofa curcas L.) Inf. Agropec., v. 26, n. 229, p. 44-78, 2005.

SOCIEDADE BRASILEIRA DA CIÊNCIA DAS PLANTAS DANINHAS - SBCPD. Procedimentos para instalação, avaliação e análise de experimentos com herbicidas. Londrina: $1995.42 \mathrm{p}$.

TEIXEIRA, L. C. Potencialidades de oleaginosas para produção de biodiesel. Inf. Agropec., v. 26, n. 229, p. 18-27, 2005.

VELINI, E. D. et al. Avaliação da seletividade da mistura de oxyfluorfen e ametryne, aplicada em pré ou pós-emergência, a dez variedades de cana-de-açúcar (cana-planta). Planta Daninha, v. 18, n. 1, p. 123-134, 2000. 